\title{
Sensitivity Analysis of Influencing Factors of Building Slope Stability Based on Orthogonal Design and Finite Element Calculation
}

\author{
RUAN Jin-kui ${ }^{1 a}$, ZHU Wei-wei ${ }^{2}$ \\ ${ }^{1}$ Yunnan Tin Industry Real Estate Development and Management Co., Ltd., 650214 Yunnan Kunming, China \\ ${ }^{2}$ Kunming University, School of Urban and Rural Construction \& Engineering Management, 650214 Yunnan Kunming, China
}

\begin{abstract}
In order to study the sensitivity of factors affecting the homogeneous building slope stability, the orthogonal test design method and shear strength reduction finite element method were used. The stability safety factor of the slope was used as the analysis index, and the range analysis of results of 18 cases were carried out. The results show that the order of sensitivity of slope stability factors is: internal friction angle, slope height, cohesion, slope angle, bulk density, elastic modulus, Poisson's ratio. The analysis results have reference significance for the design and construction of building slope projects.
\end{abstract}

\section{Introduction}

The problem of slope stability is widespread in industrial and civil buildings, roads, railways, mines, and water conservancy projects. Landslides and collapses caused by slope instability often result in huge loss of life and property. The construction slope is the artificial slope formed by the excavation or construction of the building (structure) and municipal engineering and the natural slope affecting the safety and stability of buildings in the site of construction (structure) or its surrounding area [1]. There are many factors that affect slope stability, such as slope height, slope angle, the parameters of physical and mechanical properties of rock and earth mass, construction process, role of water, weathering, earthquake and so on. The influences of these factors on the stability of the slope are certainly not the same. Therefore, it is necessary to study the sensitivity of the factors affecting the stability of the slope and provide scientific guidance for the design and construction of slope stability. At present, the research in this area is still immature, and there are few reports on the research results, and there is no consistent conclusion in the existing research results [2-8]. Through orthogonal design, the author overcomes the deficiencies of the traditional single factor analysis method and applies the shear strength reduction finite element method to calculate and statistically analyze the stability safety factors of 18 slope examples, obtain the order of sensitivity of the factors affecting stability, discuss the results of the analysis for reference by the relevant engineering technicians.

\section{Orthogonal design method}

Orthogonal design method is a kind of experimental arrangement method to study multiple factors and multiple levels. It is based on orthogonality and some representative points from comprehensive tests are selected to conduct tests. These representative points have the characteristics of "uniformly dispersed and homogeneously matched". It is a highly efficient, rapid, and economical experimental design method. In the design of orthogonal experiments, the results to be examined are called "Indicators", and the factors that may affect the "indicators" are called "Factors", and the specific conditions for each "factor" to be compared in the tests are called "Level". If a set of tests are designed, " $\mathrm{k}$ " factors are arranged, " $\mathrm{n}$ " tests are performed, and the number of levels of factors is $t 1, t 2, \ldots$, tn. If this test satisfies: (1) different levels of each factor appear the same number of times (equilibrium) in the test; (2) different combinations of any two factors appear the same number of times (orthogonality) in the test, this test is called orthogonal test. Not only the test points are evenly distributed, but also the number of tests is reduced in the orthogonal experimental design, which is a cost-effective test arrangement method. In this paper, the range analysis method is used to analyze the results of the orthogonal test, that is, to average the test results of the same level of each factor, and then obtain the range by the maximum value of the average value minus the minimum value. The greater the range is, the greater the influence of this factor on the test results.

\section{Analysis of examples}

\subsection{Calculation method}

* Corresponding author: ${ }^{\text {a } 67855517 @ q q . c o m}$ 
In this article, the slope stability safety factor is selected as the inspection index. At present, the use of the finite element method to analyze the slope stability has been greatly promoted. In the past decade or so, with the deepening of the theoretical study of the shear strength reduction method and the recognition of its advantages [9], finite element method combined with shear strength reduction has gradually become the mainstream method of slope stability finite element analysis. In his paper, this method is used to calculate the slope stability safety factor.

The shear strength reduction factor is defined as the ratio of the maximum shear strength exerted by the slope body to the actual shear stress generated by the external load in the slope with the external load remaining constant. When it is assumed that the shear strength of all slopes within the slope is exerted to the same degree, the shear strength reduction factor is defined as the overall stability factor of the slope. The basic principle of the finite element strength factor reduction method is to divide the slope strength parameters (the values of cohesion force $c$ and internal friction angle $\varphi$ ) simultaneously by a reduction factor Fs to obtain a set of new values of $c^{\prime} 、 \varphi^{\prime}$, which are then input as new material parameters and calculated. The appropriate stability judging criteria are used to determine the corresponding $F_{s}$ value is the minimum stable safety factor of the slope, and at this time the slope reaches the limit state with the occurrence of shear failure, the failure sliding surface of the slope can be obtained. Among them:

$$
c^{\prime}=\frac{c}{F_{s}}, \quad \varphi^{\prime}=\arctan \left(\frac{\tan \varphi}{F_{s}}\right) \text {. }
$$

\subsection{Calculation model}

In the calculation, ABAQUS large-scale commercial finite element software and Mohr-Coulomb failure criteria are used, and the CPE4 plane strain unit is used to establish the finite element model of the slope. The slope itself can be considered as a semi-infinite body, where the size of the slope calculation model is determined according to the optimal size given by Zhang Lu-yu et al. [9] (Figure 1). For convenience for the study, the entire slope is considered here as a homogeneous material. In the slope finite element model, the left and right boundaries limit the displacement in the $\mathrm{X}$ direction, the bottom limits the displacement in the $\mathrm{X}$ and $\mathrm{Y}$ directions, and the slope surface is free.

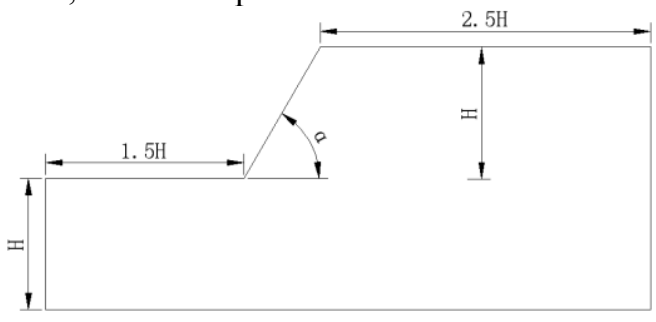

Figure 1. Calculating model of slope

\subsection{Calculation examples and results}

According to the parameters used in the finite element analysis of slope stability, seven factors such as slope height $H$, slope angle $\alpha$, cohesion $c$, internal friction angle $_{\varphi}$, bulk density $\gamma$, elastic modulus $E$, and Poisson ratio $\mu$ were selected, and three levels were selected to every factor ( Table 1), so L18 $\left(3^{7}\right)$ orthogonal array(Table 2) is used. The calculation results are also listed in Table 2. The range analysis of the calculation results is shown in Table 3, and the trend of the influence of various factors on the slope stability safety factor is shown in Figure 2.

Table 1. Factors and their levels of slope

\begin{tabular}{llllllll}
\hline & $H / \mathrm{m}$ & $\alpha /{ }^{\circ}$ & $c / \mathrm{kPa}$ & $\varphi /{ }^{\circ}$ & $\gamma / \mathrm{kN} \cdot \mathrm{m}^{-3}$ & $E / \mathrm{MPa}$ & $\mu$ \\
\hline Level 1 & 10 & 35 & 10 & 20 & 18 & 400 & 0.20 \\
Level 2 & 15 & 40 & 15 & 25 & 20 & 500 & 0.25 \\
Level 3 & 20 & 45 & 20 & 30 & 22 & 600 & 0.30 \\
\hline
\end{tabular}

Table 2. Orthogonal testing program and results of slope

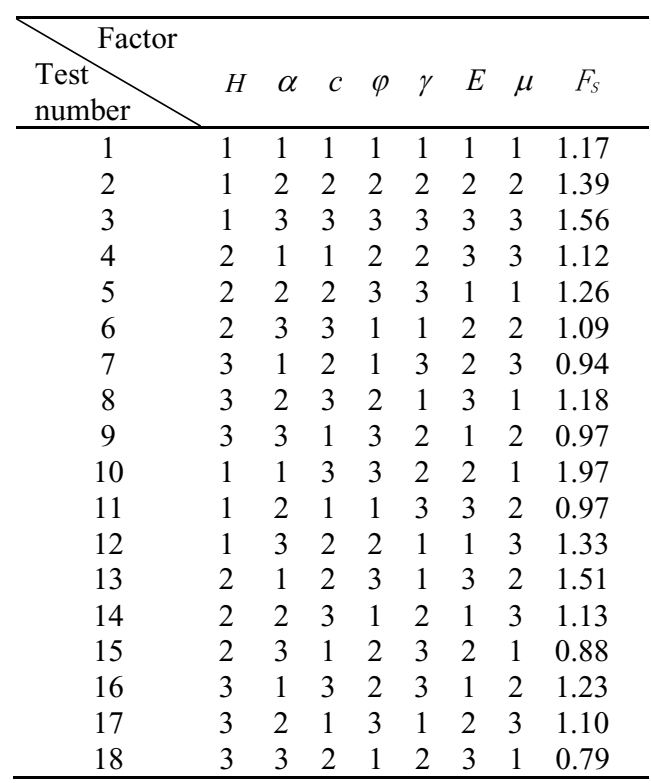

Table 3. Range analysis table

\begin{tabular}{lccccccc}
\hline & $H$ & $\alpha$ & $c$ & $\varphi$ & $\gamma$ & $E$ & $\mu$ \\
\hline Mean value 1 & 1.40 & 1.33 & 1.04 & 1.02 & 1.23 & 1.18 & 1.21 \\
Mean value 2 & 1.17 & 1.17 & 1.20 & 1.19 & 1.23 & 1.23 & 1.19 \\
Mean value 3 & 1.03 & 1.10 & 1.36 & 1.39 & 1.14 & 1.19 & 1.20 \\
Range & 0.37 & 0.23 & 0.32 & 0.37 & 0.09 & 0.05 & 0.02 \\
$\begin{array}{l}\text { The order } \\
\text { of sensitivity }\end{array}$ & 2 & 4 & 3 & 1 & 5 & 6 & 7 \\
\hline
\end{tabular}




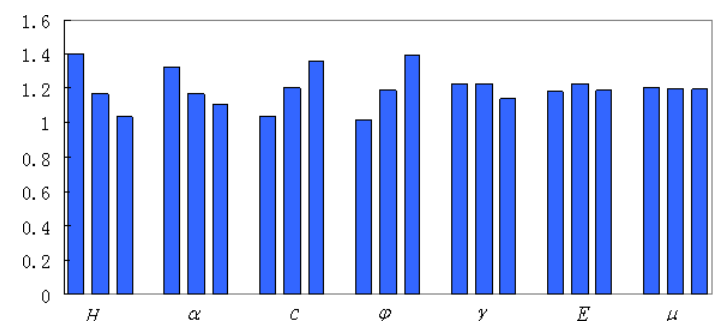

Figure 2. Impact trend to slope stability safety factor analysis of each factor

\section{Discussion of analysis results}

The above analysis results show that the internal friction angle, cohesion, and slope height are all sensitive factors affecting the stability of the slope, and the sensitivity of the three are not much different. The slope angle also has a great influence on the stability, and others are secondary influencing factors. From the point of view of design and construction, it is of great significance to stabilize the slopes by slowing slopes, avoiding deep cuts of slopes, and strengthening drainage. The existing research results on the sensitivity of slope stability parameters are not the same. For example, the analysis result of literature [2] is: $c>\varphi>\gamma$; the result of the literature [3] is: $c>\gamma>\varphi>\alpha>H$; it is recognized that in literature [7] $\varphi>c>\gamma>H>\mu>\alpha$ and so on. The conclusions obtained by the author are not the same as those in the literature, which may be related to factors such as the test arrangement methods, safety factor calculation method, and the calculation range of the slope model. For example, the author tried to increase the calculation range of the slope (larger sizes are extended in left, right, and bottom parts). This will result in the development of multiple sets of sliding surfaces in the slope, and it is difficult to obtain a single ideal sliding surface, thereby affecting the discrimination of safety factor. Another example is that in some studies the foundation part is assigned higher mechanical parameters, which will also affect the calculation of the safety factor, ad such treatment is not fully supported and is not representative except for the analysis to specific slope with a high intensity of slope of foundation. Since the calculation model and the calculation methods are not mentioned in literature, and the analysis factors chosen are not the same, it is not easy to analyze the clear reasons for the differences.

\section{Conclusions}

(1) The order of sensitivity of the factors affecting the slope stability obtained in this paper is $\varphi>H>c>\alpha>\gamma>$ $E>\mu$. It can be seen that the shear strength index, slope height and slope angle are the most sensitive to the slope stability, and the bulk density effect is secondary, while the remaining factors have little effect.

(2) In designing and constructing the occurrence of high slope due to deep excavation should be avoided and the slope should be slowed. In the slope stability analysis, the cohesion force $c$ and internal friction angle $\varphi$ should take the average of small values of the test results, and the bulk density $Y$ can take the average value. In the numerical analysis, if the test data are absent, empirical values can be used to calculate the elastic modulus and Poisson's ratio, which have little effect on the results.

The study of sensitivity of the factors affecting slope stability is very complicated. In this article, the homogeneous slope with strength reduction finite element method being adopted to calculate the size of a specific model is analyzed. In actual construction projects, the effects of groundwater, surface water, seismic loads, slope load, excavation methods, and heterogeneity may also be considered according to the specific circumstances.

\section{Acknowledgements}

This research was funded by Yunnan Applied Basic Research Projects (2016FD108).

\section{References}

1. Ministry of Housing and Urban-Rural Development of the PRC, Technical code for building slope engineering (CAB Press, Beijing, 2013)

2. ZHANG Shao-hong. Journal of Water Resources and Architectural Engineering 1,40-42. (2003)

3. LIU Li-peng, YU hong-jie, CHEN Qi. Geotechnical Engineering Technique 22,123-126. (2008)

4. TANG Fen, ZHENG Ying-ren. Journal of Highway and Transportation Research and Development 25,39-44. (2008)

5. DAI Zhi-ren, ZHANG Sha-sha, XIE Yong-li. Subgrade Engineering,100-102. (2010)

6. WANG Yi-ping, ZHOU Rong-chao. Journal of Yancheng Institute of Technology Natural Science Edition 20,67-69. (2007)

7. XIA Yuan-you, XIONG Hai-feng. Chinese Journal of Rock Mechanics and Engineering 23,2703-2707. (2004)

8. ZHANG Xu-hui, GONG Xiao-nan, XU Ri-qing. China Journal of Highway and Transportation 16,36-39. (2003)

9. ZHANG Lu-yu, ZHENG Ying-ren, ZHAO Shang-yi, et al. SHUILI XUEBAO 21-27. (2003) 